\title{
Synthesis, Spectroscopy, and Theoretical Details of Uranyl Schiff Base Coordination Complexes
}

Bonnie E. Klamm, Cory J. Windorff, Cristian Celis-Barros, Matthew L. Marsh, and Thomas E. Albrecht-Schmitt*

Department of Chemistry and Biochemistry, Florida State University, 95 Chieftan Way, RM. 118 DLC, Tallahassee, Florida 32306, United States.

*talbrechtschmitt@fsu.edu

Table of Contents

Page

NMR Spectra S2-S5

FTIR Spectra .S6-S7

UV/vis/NIR Spectra and Image. S8

Cyclic Voltammetry S9-S13

Computational Details. S14- S17

Crystallographic Details S18-S19 


\section{NMR Spectra}

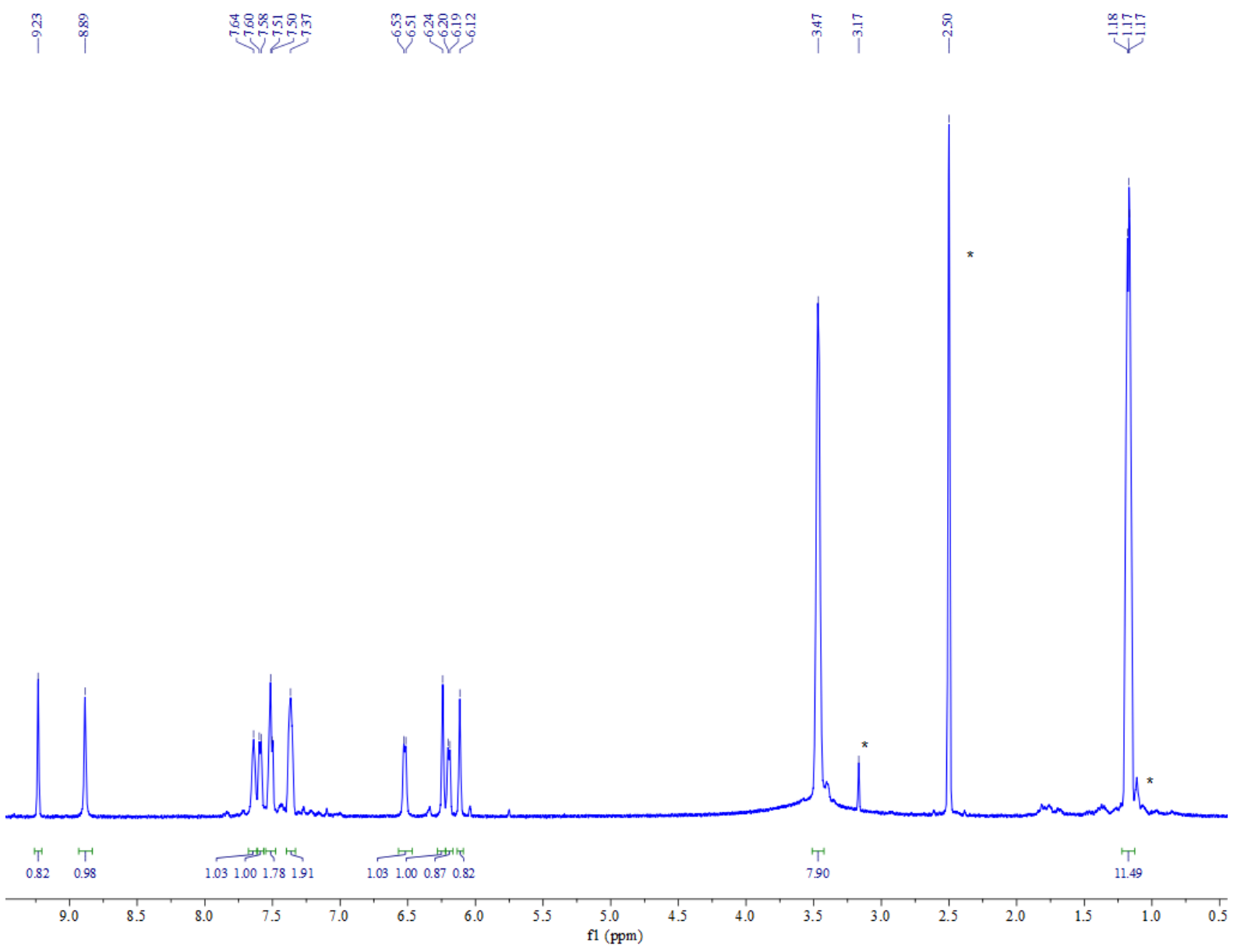

Figure S1. ${ }^{1} \mathrm{H}$ NMR spectrum of $\mathbf{U O}_{2} \mathbf{L}(\mathbf{M e O H})$ in DMSO at $298 \mathrm{~K}$ with $*$ denoting solvent resonance. 


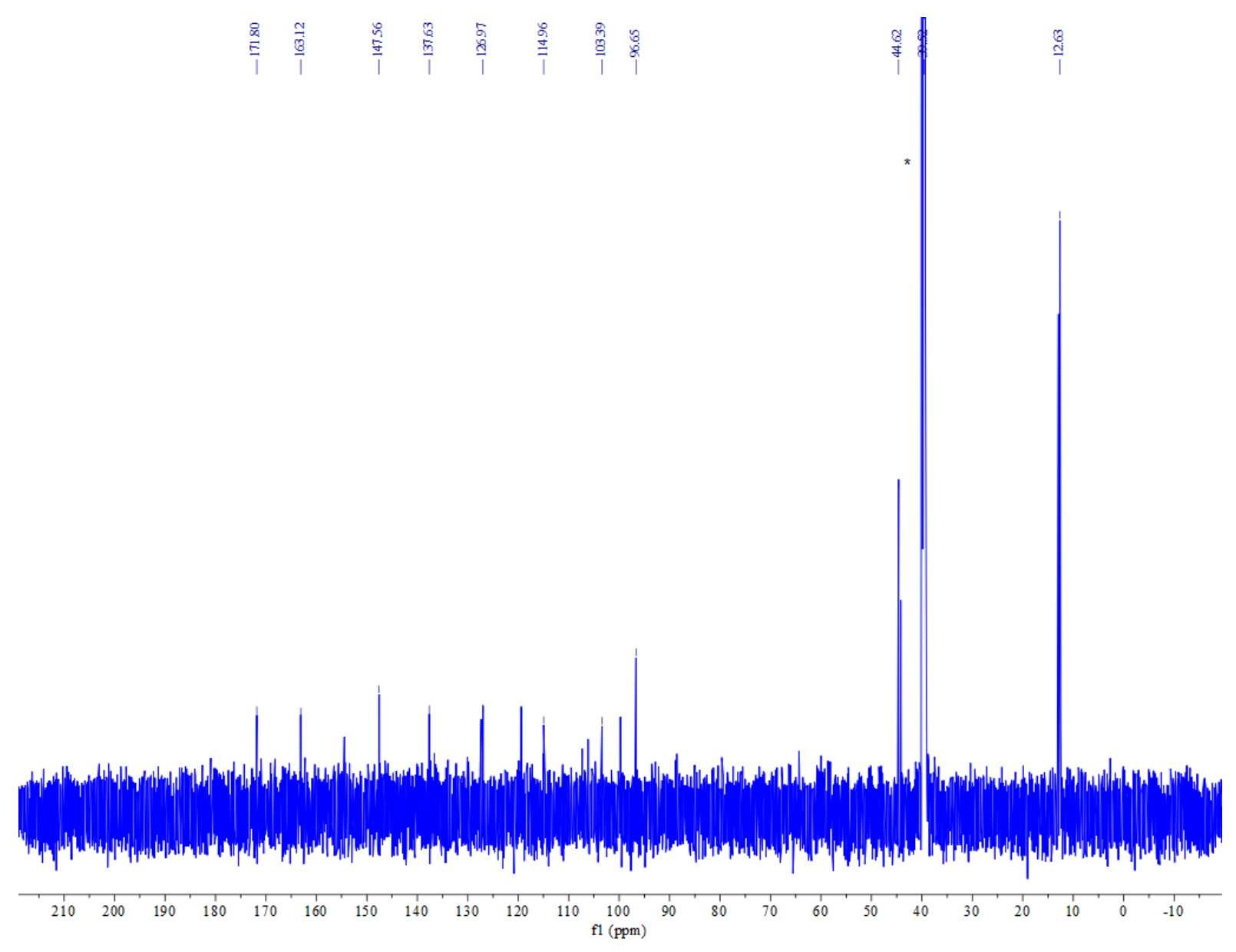

Figure S2. ${ }^{13} \mathrm{C}\left\{{ }^{1} \mathrm{H}\right\}$ NMR spectrum of $\mathbf{U O}_{2} \mathbf{L}(\mathbf{M e O H})$ in DMSO at $298 \mathrm{~K}$ with $*$ denoting solvent resonance. 


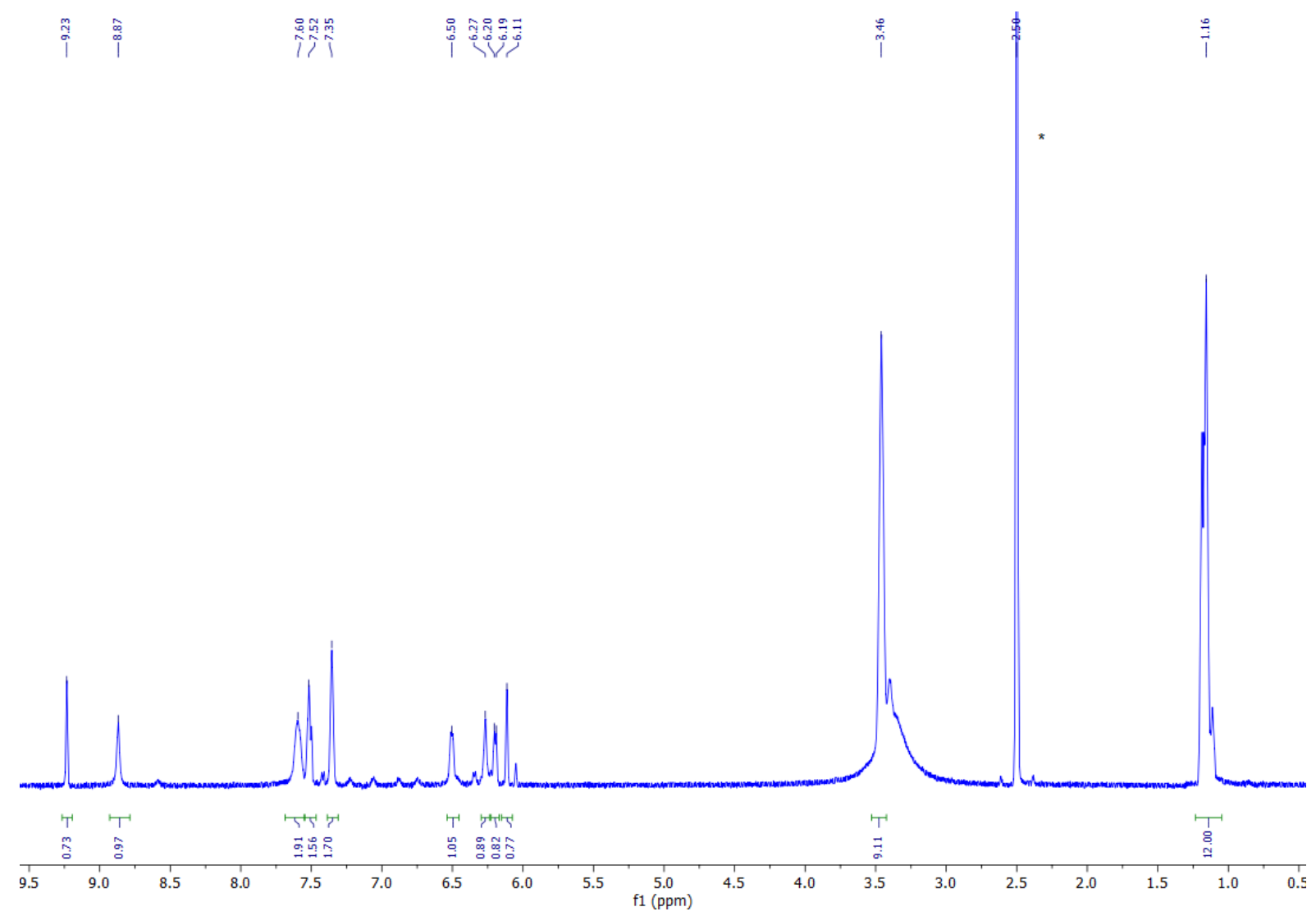

Figure S3. ${ }^{1} \mathrm{H}$ NMR spectrum of $\mathbf{U O}_{2} \mathbf{C l}_{2}\left(\mathbf{H}_{2} \mathbf{L}\right)$ in DMSO at $298 \mathrm{~K}$ with $*$ denoting solvent resonance. 


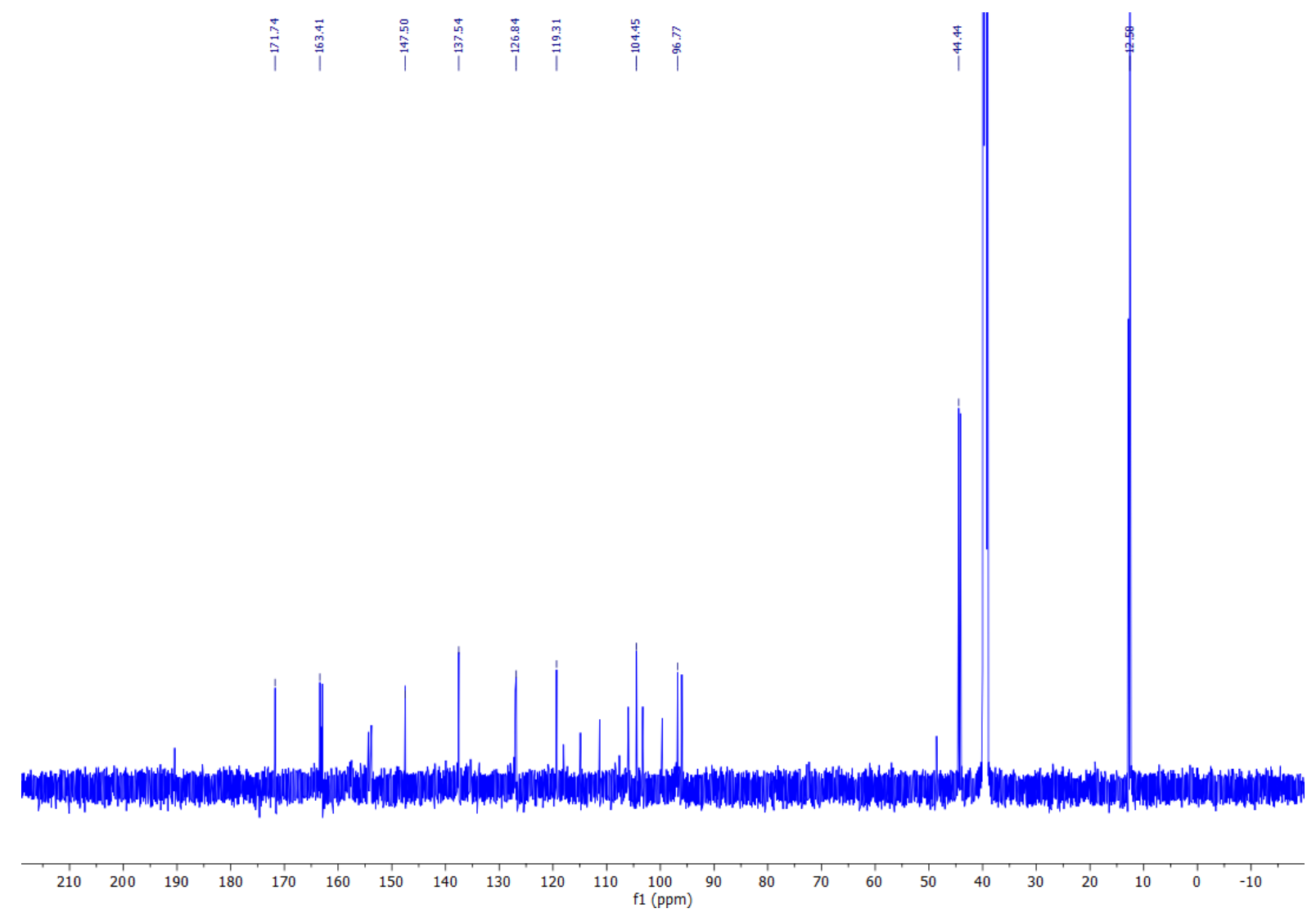

Figure S4. ${ }^{13} \mathrm{C}\left\{{ }^{1} \mathrm{H}\right\}$ NMR spectrum of $\mathbf{U O}_{2} \mathbf{C l}_{2}\left(\mathbf{H}_{2} \mathbf{L}\right)$ in DMSO at $298 \mathrm{~K}$ with $*$ denoting solvent resonance. 


\section{FTIR Spectra}

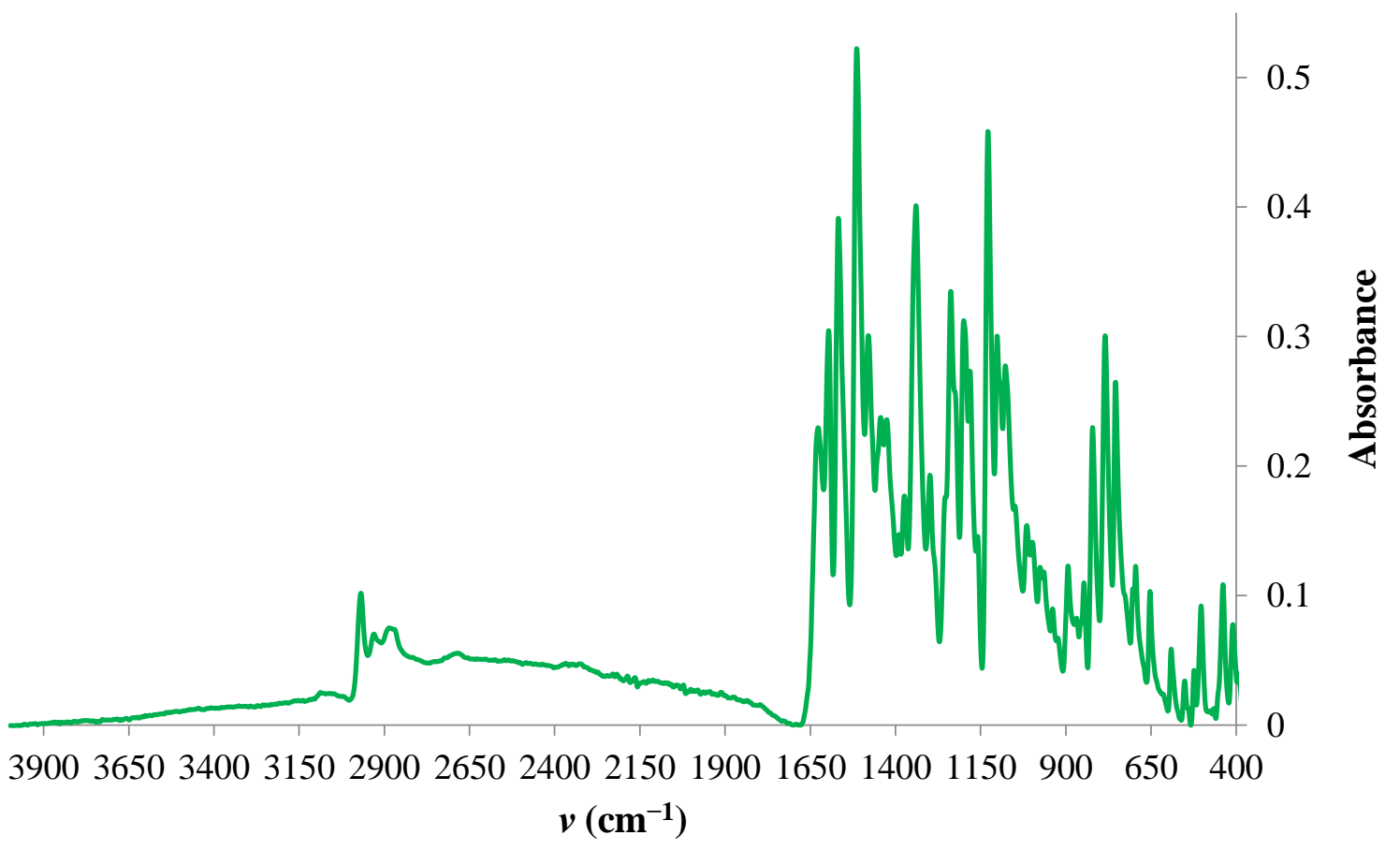

Figure S5. Solid state infrared spectrum of $\mathbf{H}_{2} \mathbf{L}$, absorption peaks, $v=3083 \mathrm{vw}, 2979 \mathrm{w}$, 2930w, 2885w, 2688br, 1630s, 1596s, 1568s, 1514s, 1481s, 1446m, 1427m, 1392w, 1375m, $1341 \mathrm{~s}, 1300 \mathrm{~m}, 1238 \mathrm{~s}, 1201 \mathrm{~s}, 1181 \mathrm{~s}, 1165 \mathrm{sh}, 1129 \mathrm{~s}, 1102 \mathrm{~s}, 1079 \mathrm{~s}, 1057 \mathrm{sh}, 1017 \mathrm{~m}, 1001 \mathrm{~m}$, 977m, 972m, 940w, 895m, 877w, 848m, 821s, 786s, 755s, 704sh, 695m, 654m, 592m, 552w, 524w, 504m, 439m, $410 \mathrm{~m} \mathrm{~cm}^{-1}$.

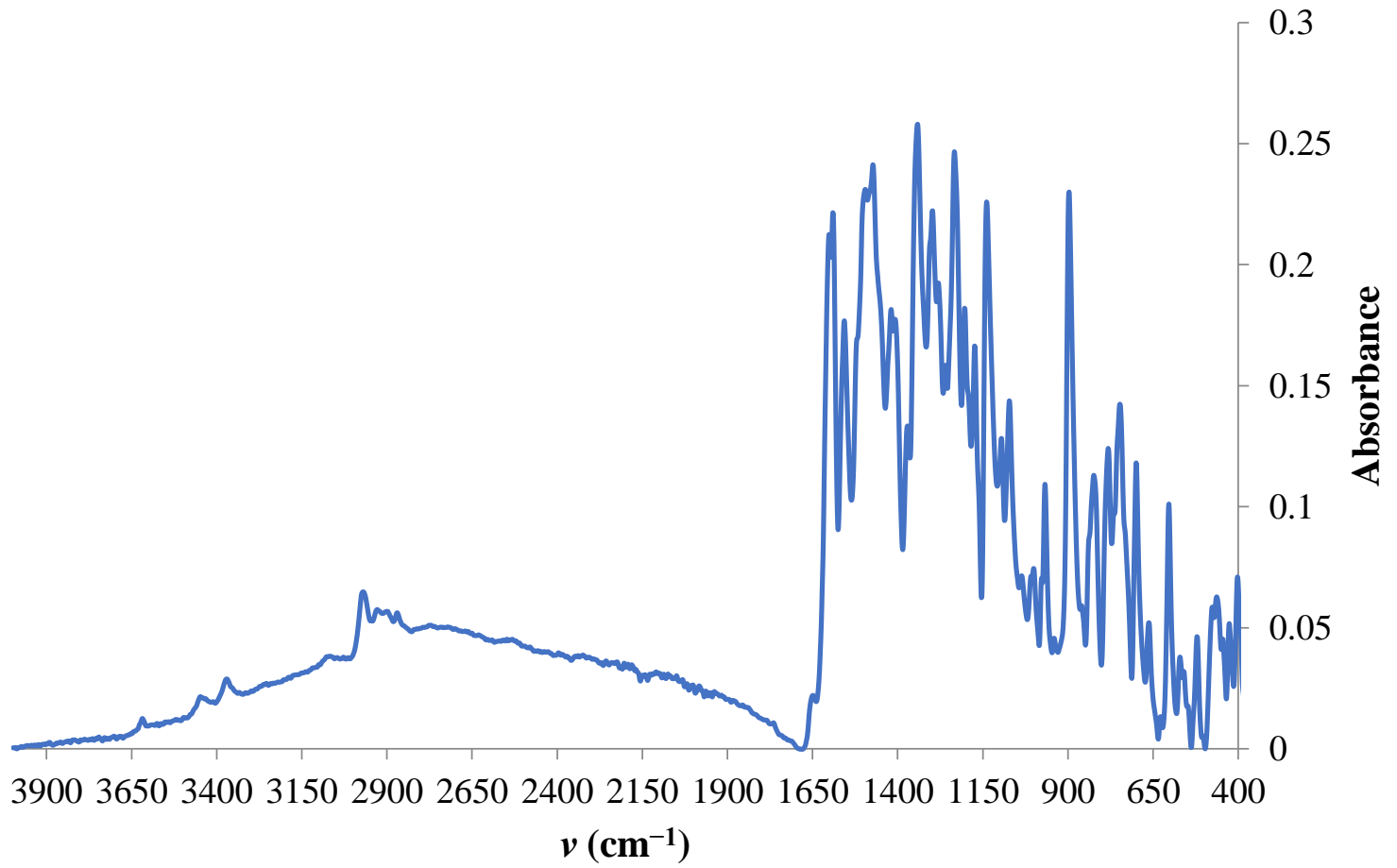

Figure S6. Solid state infrared spectrum of $\mathrm{UO}_{2} \mathbf{L}(\mathbf{M e O H})$. 


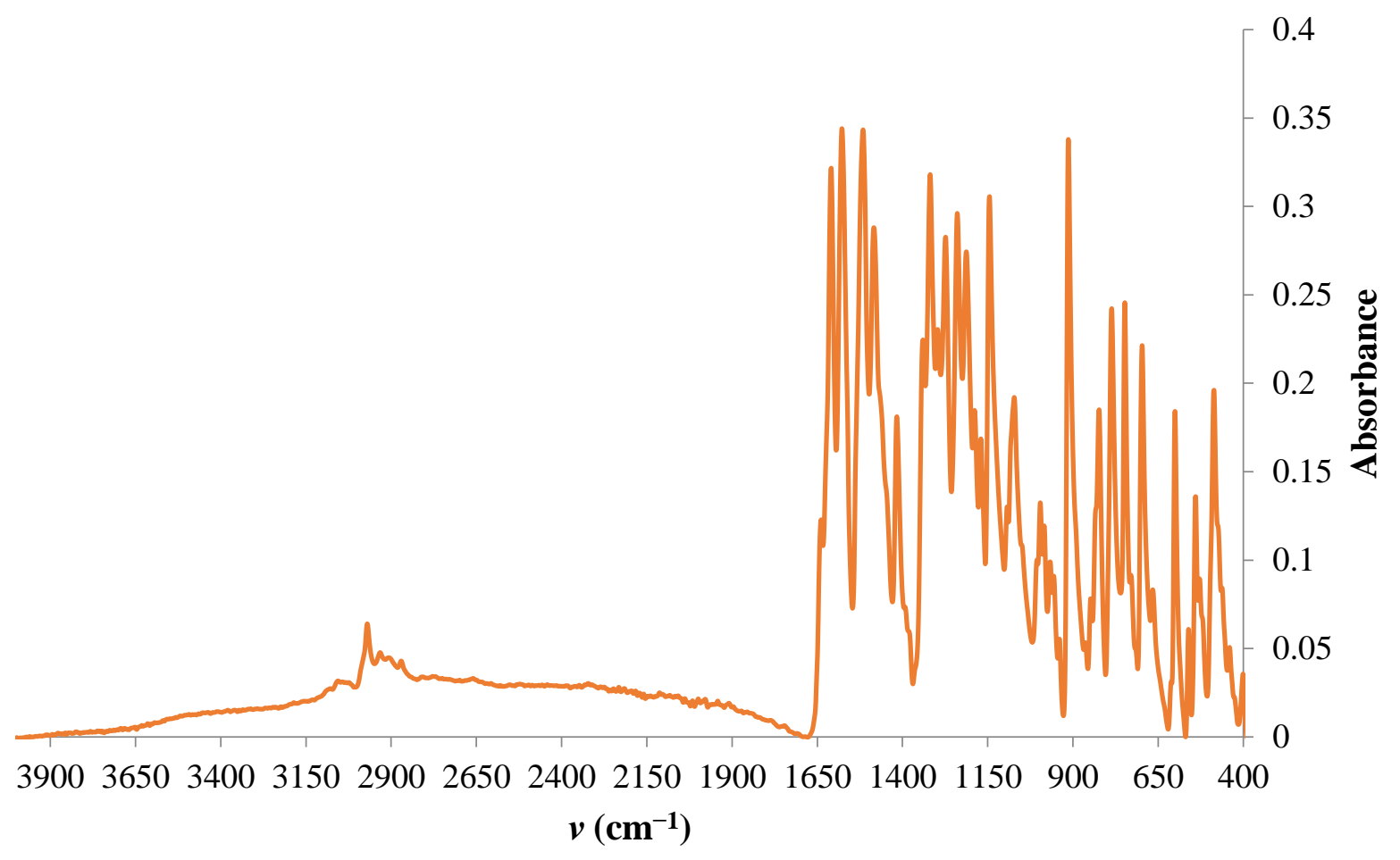

Figure S7. Solid state infrared spectrum of $\mathrm{UO}_{2} \mathrm{Cl}_{2}\left(\mathrm{H}_{2} \mathbf{L}\right)$. 


\section{UV/vis/NIR Spectra and Image}

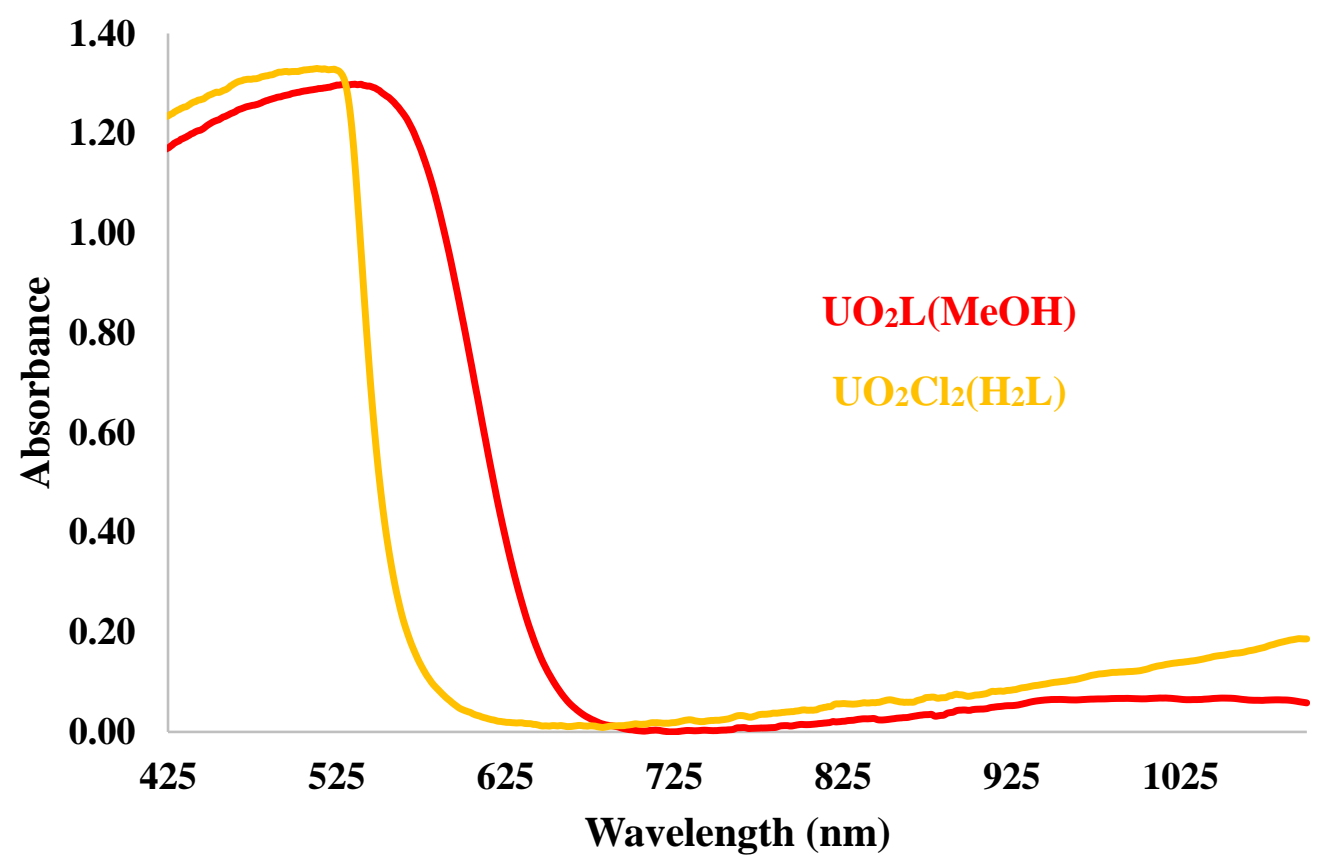

Figure S8. Solid state UV/vis/NIR absorption spectra of $\mathrm{UO}_{2} \mathbf{L}(\mathbf{M e O H})$, and $\mathbf{U O}_{2} \mathrm{Cl}_{2}\left(\mathbf{H}_{2} \mathbf{L}\right)$.

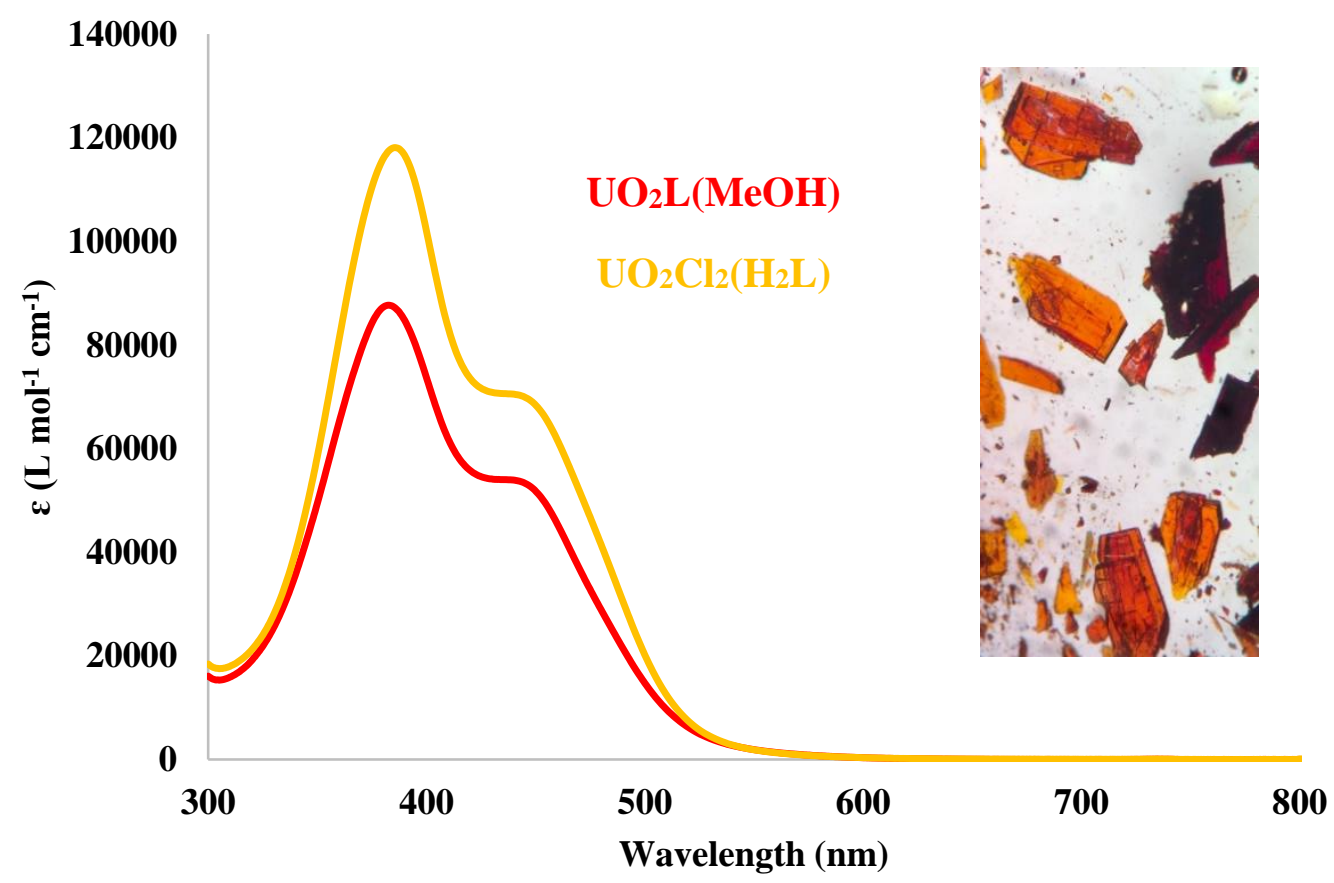

Figure S9 Solution phase UV/vis/NIR absorption spectra of $\mathrm{UO}_{2} \mathbf{L}(\mathbf{M e O H})$ and $\mathrm{UO}_{2} \mathrm{Cl}_{2}\left(\mathrm{H}_{2} \mathbf{L}\right)$ in DMSO at $298 \mathrm{~K}$ and photograph of complexes. 


\section{Cyclic Voltammetry}

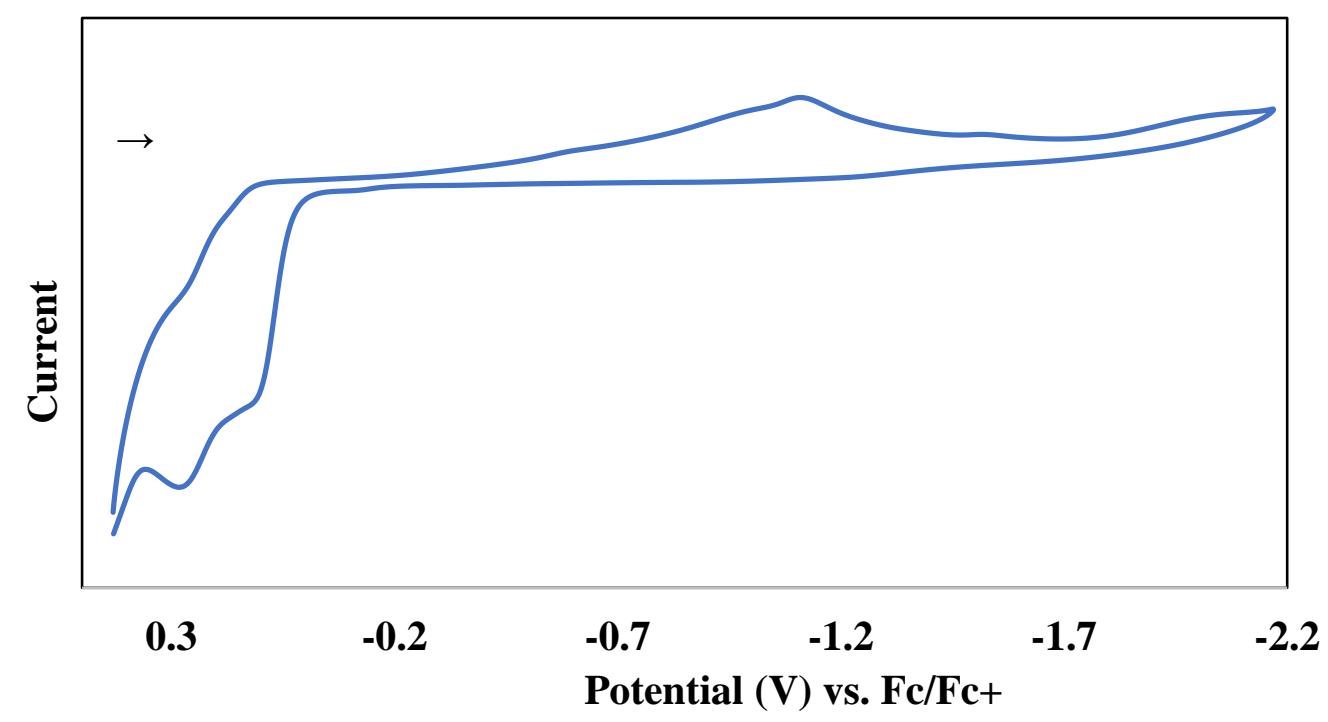

Figure S10. Cyclic voltammogram of $\mathbf{H}_{2} \mathbf{L}$ at a concentration of $5 \mathrm{mM}$ in $0.1 \mathrm{M}$ [TBA] $\left.\mathrm{PF}_{6}\right]$ and DMSO at scan rate $0.1 \mathrm{~V} / \mathrm{s}$.

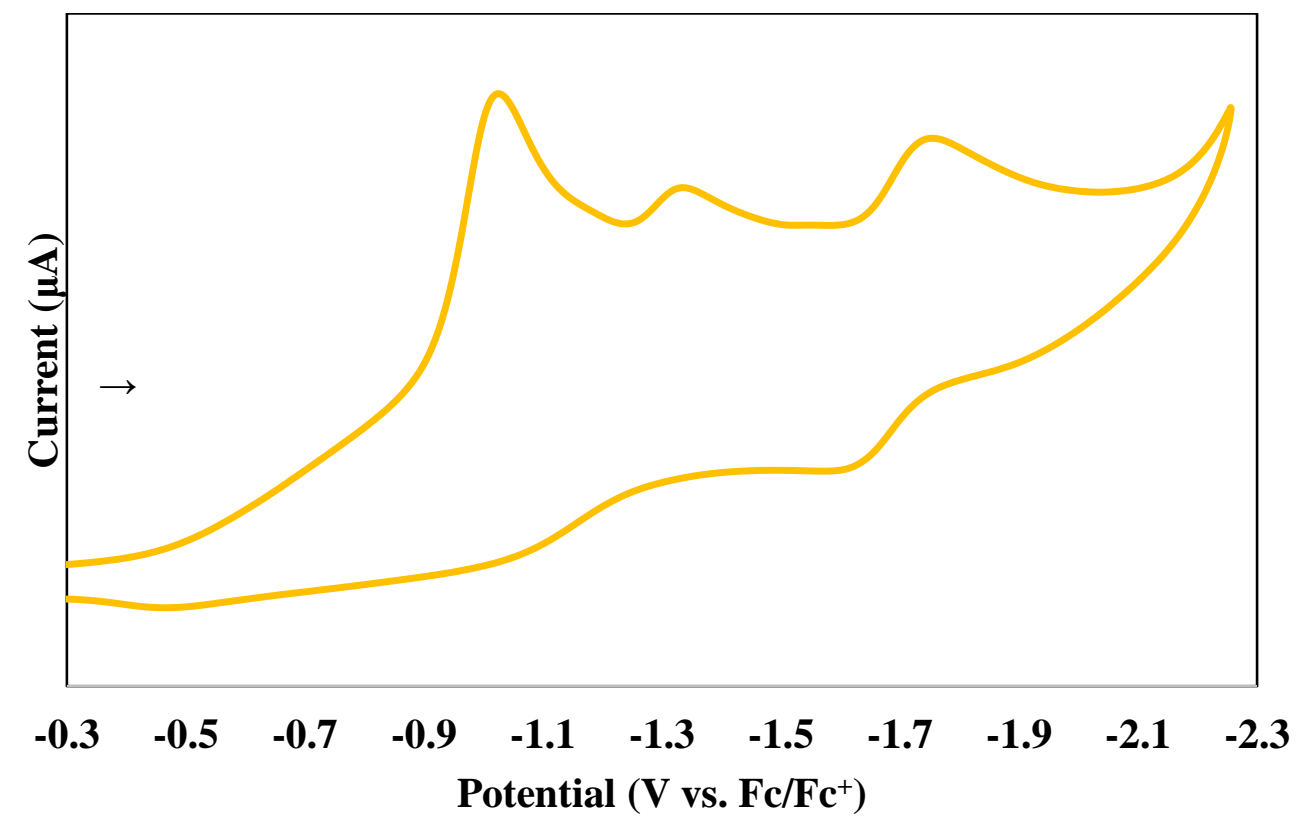

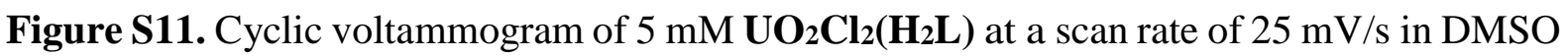
with $0.1 \mathrm{M}[\mathrm{TBA}]\left[\mathrm{PF}_{6}\right]$ supporting electrolyte. 


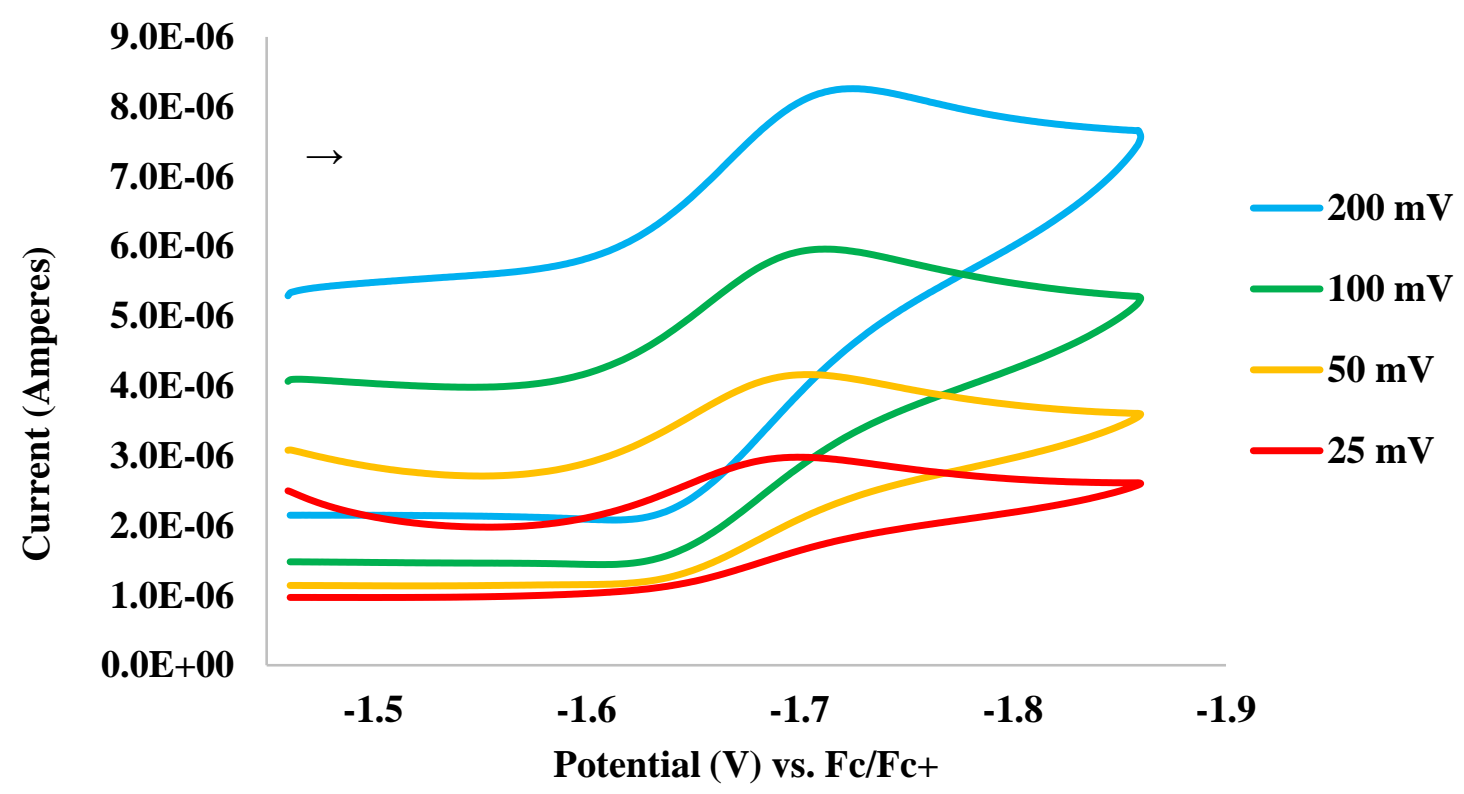

Figure S12. Cyclic voltammogram of $4 \mathrm{mM} \mathbf{U O}_{2} \mathbf{C l}_{2}\left(\mathbf{H}_{2} \mathbf{L}\right)$ at a scan rate of $\mathrm{v}=25,50,100$, and $200 \mathrm{mV} / \mathrm{sec}$ in DMSO with $0.1 \mathrm{M}$ [TBA] $\left[\mathrm{PF}_{6}\right]$ supporting electrolyte. 


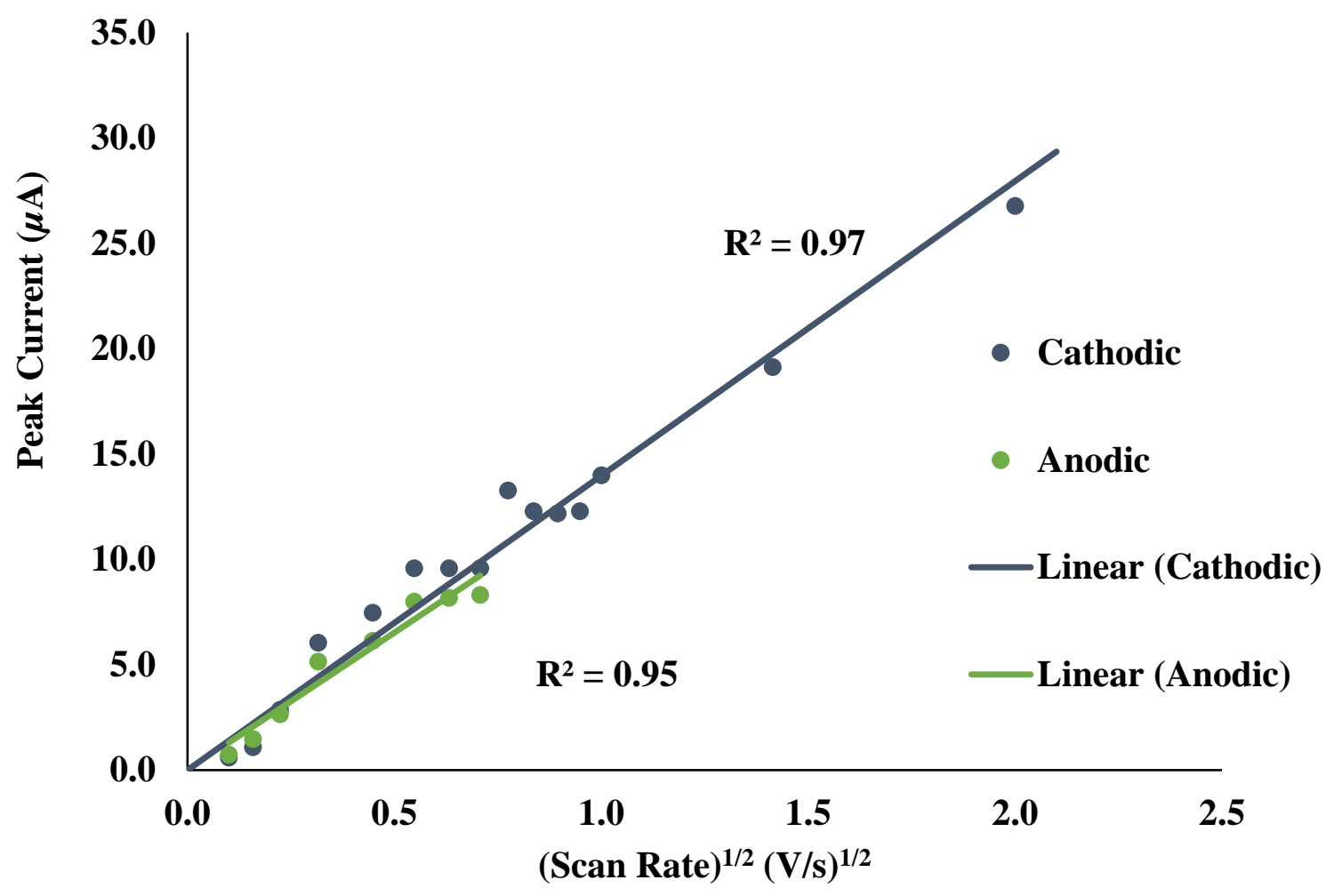

Figure S13. A plot of the (scan rate) ${ }^{1 / 2}$ and the absolute value of cathodic (blue) and anodic (green) peak currents show a linear relationship, allowing a calculation of $\mathrm{D}=2.56 \pm 0.21 \times$ $10^{-7} \mathrm{~cm}^{2} / \mathrm{s}$ for $\mathbf{U O}_{2} \mathbf{L}(\mathbf{M e O H})$. 
Table S1. Baseline corrections and current data for $\mathrm{UO}_{2} \mathbf{L}(\mathbf{M e O H})$.

\begin{tabular}{|c|c|c|c|c|c|c|c|}
\hline $\begin{array}{l}\text { Scan } \\
\text { Rate } \\
(\mathrm{V} / \mathrm{s})\end{array}$ & $\begin{array}{c}\text { Square } \\
\text { Root } \\
\text { Scan } \\
\text { Rate } \\
(\mathrm{V} / \mathrm{s})^{1 / 2}\end{array}$ & $\begin{array}{c}\text { Baseline } \\
(\mu \mathrm{A})\end{array}$ & $\begin{array}{c}\text { Cathodic } \\
\text { Peak } \\
\text { Current } \\
(\mu \mathrm{A})\end{array}$ & $\begin{array}{c}\text { Corrected } \\
\text { Cathodic } \\
\text { Current } \\
(\mu \mathrm{A})\end{array}$ & $\begin{array}{c}\text { Anodic } \\
\text { Peak } \\
\text { Current } \\
(\mu \mathrm{A})\end{array}$ & $\begin{array}{c}\text { Corrected } \\
\text { Anodic } \\
\text { Current } \\
(\mu \mathrm{A})\end{array}$ & $I_{\mathrm{pd}} / I_{\mathrm{pa}}$ \\
\hline 0.01 & 0.1 & 1.42 & 2.01 & 0.59 & -0.69 & 0.73 & 0.81 \\
\hline 0.025 & 0.158114 & 2.16 & 3.24 & 1.08 & -0.69 & 1.47 & 0.74 \\
\hline 0.05 & 0.223607 & 2.84 & 5.70 & 2.86 & -0.18 & 2.66 & 1.08 \\
\hline 0.1 & 0.316228 & 3.81 & 9.86 & 6.05 & 1.34 & 5.15 & 1.17 \\
\hline 0.2 & 0.447214 & 3.74 & 11.20 & 7.47 & 2.39 & 6.13 & 1.22 \\
\hline 0.3 & 0.547723 & 4.22 & 13.80 & 9.58 & 3.78 & 8.00 & 1.20 \\
\hline 0.4 & 0.632456 & 4.22 & 13.80 & 9.58 & 3.95 & 8.17 & 1.17 \\
\hline 0.5 & 0.707107 & 4.22 & 13.80 & 9.58 & 4.09 & 8.31 & 1.15 \\
\hline 0.6 & 0.774597 & 4.22 & 17.50 & 13.28 & 5.32 & 9.54 & 1.39 \\
\hline 0.7 & 0.83666 & 4.22 & 16.50 & 12.28 & 5.30 & 9.52 & 1.29 \\
\hline 0.8 & 0.894427 & 4.22 & 16.4 & 12.18 & 5.31 & 9.53 & 1.28 \\
\hline 0.9 & 0.948683 & 4.22 & 16.5 & 12.28 & 5.30 & 9.52 & 1.29 \\
\hline 1 & 1 & 3.4 & 17.4 & 14 & 5.74 & 9.14 & 1.53 \\
\hline 2 & 1.414214 & 3.37 & 22.5 & 19.13 & 8.26 & 11.63 & 1.64 \\
\hline 4 & 2 & 3.925 & 30.7 & 26.775 & 12.3 & 16.225 & 1.65 \\
\hline
\end{tabular}


Table S2. Kinetic data for $\mathrm{UO}_{2} \mathrm{~L}(\mathbf{M e O H})$

\begin{tabular}{|c|c|c|}
\hline Scan Rate $(\mathrm{V} / \mathrm{s})$ & Reversibility $\Delta E_{\mathrm{p}}(\mathrm{mV})$ & Nicholson Parameter $(\psi)$ \\
\hline 0.01 & 62 & 3.15 \\
\hline 0.025 & 67 & 0.82 \\
\hline 0.05 & 89 & 0.36 \\
\hline 0.1 & 120 & 0.3 \\
\hline 0.2 & 127 & 0.22 \\
\hline 0.3 & 150 & 0.235 \\
\hline 0.4 & 146 & 0.193 \\
\hline 0.5 & 159 & 0.188 \\
\hline 0.6 & 168 & 0.18 \\
\hline 0.7 & 172 & 0.188 \\
\hline 0.8 & 168 & 0.18 \\
\hline 0.9 & 172 & 0.16 \\
\hline 1.0 & 182 & \\
\hline & & \\
\hline
\end{tabular}




\section{Theory Analysis}

Table S3. Decomposition of the multiconfigurational ground states using the SO-PT2 $(6,10)$ wave functions. Ligand orbitals $p_{\sigma}$ and $p_{\pi}$ refer to the $2 p$ oxygen orbitals that mix with the $5 f$ orbitals are depicted in red.

\begin{tabular}{|c|c|c|c|c|c|c|}
\hline State & \multicolumn{2}{|c|}{$\mathrm{UO}_{2}{ }^{2+}$} & \multicolumn{2}{|c|}{$\mathbf{U O}_{2} \mathbf{C l}_{2}\left(\mathbf{H}_{2} \mathbf{L}\right)$} & \multicolumn{2}{c|}{$\mathbf{U O}_{2}(\mathbf{M e O H}) \mathbf{L}$} \\
\hline \multirow{3}{*}{$\Sigma_{\mathrm{g}}$} & $79 \%$ & $p_{\pi}^{4} p_{\sigma}^{2} f_{\delta}^{0} f_{\varphi}^{0} f_{\pi}^{0} f_{\sigma}^{0}$ & $76 \%$ & $p_{\pi}^{4} p_{\sigma}^{2} f_{\delta}^{0} f_{\varphi}^{0} f_{\pi}^{0} f_{\sigma}^{0}$ & $73 \%$ & $p_{\pi}^{4} p_{\sigma}^{2} f_{\delta}^{0} f_{\varphi}^{0} f_{\pi}^{0} f_{\sigma}^{0}$ \\
\cline { 2 - 7 } & $13 \%$ & $p_{\pi}^{3} p_{\sigma}^{2} f_{\delta}^{0} f_{\varphi}^{0} f_{\pi}^{1} f_{\sigma}^{0}$ & $14 \%$ & $p_{\pi}^{3} p_{\sigma}^{2} f_{\delta}^{0} f_{\varphi}^{0} f_{\pi}^{1} f_{\sigma}^{0}$ & $17 \%$ & $p_{\pi}^{3} p_{\sigma}^{2} f_{\delta}^{0} f_{\varphi}^{0} f_{\pi}^{1} f_{\sigma}^{0}$ \\
\cline { 2 - 7 } & $3 \%$ & $p_{\pi}^{4} p_{\sigma}^{1} f_{\delta}^{0} f_{\varphi}^{0} f_{\pi}^{0} f_{\sigma}^{1}$ & $4 \%$ & $p_{\pi}^{4} p_{\sigma}^{1} f_{\delta}^{0} f_{\varphi}^{0} f_{\pi}^{0} f_{\sigma}^{1}$ & $5 \%$ & $p_{\pi}^{4} p_{\sigma}^{1} f_{\delta}^{0} f_{\varphi}^{0} f_{\pi}^{0} f_{\sigma}^{1}$ \\
\hline
\end{tabular}

Table S4. NBOs involving the $\mathrm{UO}_{2}{ }^{2+}$ ion, their compositions in terms of natural hybrid orbitals, assignment, and occupation numbers derived from PBE0/TZVP wavefunctions

\begin{tabular}{|c|c|c|c|c|c|c|c|}
\hline \multirow{2}{*}{$\mathrm{NBO}$} & \multirow{2}{*}{$\begin{array}{l}\text { Bond } \\
\text { type }\end{array}$} & \multicolumn{2}{|l|}{$\mathrm{UO}_{2}{ }^{2+}$} & \multicolumn{2}{|l|}{$\mathrm{UO}_{2} \mathrm{Cl}_{2}\left(\mathrm{H}_{2} \mathrm{~L}\right)$} & \multicolumn{2}{|l|}{$\mathrm{UO}_{2} \mathrm{~L}(\mathrm{MeOH})$} \\
\hline & & NHOs & Occ & NHOs & Occ & NHOs & Occ \\
\hline $\mathrm{BD}(1)$ & $\pi$ & $\begin{array}{c}76 \% \mathrm{O}_{\mathrm{yl}}(p)+24 \% \\
\mathrm{U}(f d)\end{array}$ & 1.99 & $\begin{array}{c}79 \% \mathrm{O}_{\mathrm{yl}}(p)+21 \% \\
\mathrm{U}(f d)\end{array}$ & 1.99 & $\begin{array}{c}79 \% \mathrm{O}_{\mathrm{yl}}(p)+21 \% \\
\mathrm{U}(f d)\end{array}$ & 1.99 \\
\hline $\mathrm{BD}(2)$ & $\pi$ & $\begin{array}{c}76 \% \mathrm{O}_{\mathrm{yl}}(p)+24 \% \\
\mathrm{U}(f d)\end{array}$ & 1.99 & $\begin{array}{c}79 \% \mathrm{O}_{\mathrm{yl}}(p)+21 \% \\
\mathrm{U}(f d)\end{array}$ & 1.99 & $\begin{array}{c}80 \% \mathrm{O}_{\mathrm{yl}}(p)+20 \% \\
\mathrm{U}(f d)\end{array}$ & 1.99 \\
\hline $\mathrm{BD}(3)$ & $\sigma$ & $\begin{array}{c}64 \% \mathrm{O}_{\mathrm{yl}}\left(s p^{3}\right)+ \\
36 \% \mathrm{U}(f d)\end{array}$ & 1.90 & $\begin{array}{c}73 \% \mathrm{O}_{\mathrm{yl}}\left(s p^{3}\right)+27 \% \\
\mathrm{U}(f d)\end{array}$ & 1.92 & $\begin{array}{c}73 \% \mathrm{O}_{\mathrm{yl}}\left(s p^{3}\right)+27 \% \\
\mathrm{U}(f d)\end{array}$ & 1.92 \\
\hline $\mathrm{BD}(4)$ & $\pi$ & $\begin{array}{c}76 \% \mathrm{O}_{\mathrm{yl}}(p)+24 \% \\
\mathrm{U}(f d)\end{array}$ & 1.99 & $\begin{array}{c}79 \% \mathrm{O}_{\mathrm{yl}}(p)+21 \% \\
\mathrm{U}(f d)\end{array}$ & 1.99 & $\begin{array}{c}80 \% \mathrm{O}_{\mathrm{yl}}(p)+20 \% \\
\mathrm{U}(f d)\end{array}$ & 1.99 \\
\hline $\mathrm{BD}(5)$ & $\pi$ & $\begin{array}{c}76 \% \mathrm{O}_{\mathrm{yl}}(p)+24 \% \\
\mathrm{U}(f d)\end{array}$ & 1.99 & $\begin{array}{c}79 \% \mathrm{O}_{\mathrm{yl}}(p)+21 \% \\
\mathrm{U}(f d)\end{array}$ & 1.99 & $\begin{array}{c}79 \% \mathrm{O}_{\mathrm{yl}}(p)+21 \% \\
\mathrm{U}(f d)\end{array}$ & 1.98 \\
\hline $\mathrm{BD}(6)$ & $\sigma$ & $\begin{array}{c}64 \% \mathrm{O}_{\mathrm{yl}}\left(\mathrm{s} p^{6}\right)+ \\
36 \% \mathrm{U}(f d)\end{array}$ & 1.90 & $\begin{array}{c}73 \% \mathrm{O}_{\mathrm{yl}}\left(s p^{3}\right)+27 \% \\
\mathrm{U}(f d)\end{array}$ & 1.91 & $\begin{array}{c}75 \% \mathrm{O}_{\mathrm{yl}}\left(s p^{3}\right)+25 \% \\
\mathrm{U}(f d)\end{array}$ & 1.92 \\
\hline LV(1) & NB & $\begin{array}{c}\mathrm{U}(87 \% 7 s+13 \% \\
6 d)\end{array}$ & 0.04 & $\mathrm{U}(100 \% 6 d)$ & 0.37 & $\mathrm{U}(58 \% 5 f+42 \% 6 d)$ & 0.38 \\
\hline
\end{tabular}




\begin{tabular}{|c|c|c|c|c|c|c|c|}
\hline $\mathrm{LV}(2)$ & NB & $\mathrm{U}(100 \% 5 f)$ & 0.00 & $\begin{array}{c}\mathrm{U}(48 \% 5 f+35 \% 7 s+ \\
17 \% 6 d)\end{array}$ & 0.31 & $\mathrm{U}(84 \% 6 d+16 \% 5 f)$ & 0.31 \\
\hline $\mathrm{LV}(3)$ & NB & $\mathrm{U}(100 \% 5 f)$ & 0.00 & $\mathrm{U}(100 \% 5 f)$ & 0.22 & $\begin{array}{c}\mathrm{U}(76 \% 7 s+14 \% 6 d+ \\
10 \% 5 f\end{array}$ & 0.20 \\
\hline LV(4) & NB & $\mathrm{U}(100 \% 5 f)$ & 0.00 & $\mathrm{U}(66 \% 6 d+34 \% 7 s)$ & 0.20 & $\begin{array}{c}\mathrm{U}(42 \% 6 d+39 \% 5 f+ \\
19 \% 7 s)\end{array}$ & 0.20 \\
\hline $\mathrm{LV}(5)$ & NB & $\mathrm{U}(100 \% 5 f)$ & 0.00 & $\begin{array}{c}\mathrm{U}(51 \% 5 f+30 \% 7 s+ \\
19 \% 6 d)\end{array}$ & 0.17 & $\begin{array}{c}\mathrm{U}(78 \% 5 f+18 \% 6 d+ \\
4 \% 7 s)\end{array}$ & 0.15 \\
\hline LV(6) & & & 0.00 & $\mathrm{U}(100 \% 5 f)$ & 0.13 & $\mathrm{U}(84 \% 5 f+16 \% 6 d)$ & 0.07 \\
\hline $\mathrm{LV}(7)$ & & & 0.00 & $\begin{array}{c}\mathrm{U}(75 \% 5 f+20 \% 6 d+ \\
5 \% 7 p)\end{array}$ & 0.01 & $\mathrm{U}(100 \% 5 f)$ & 0.03 \\
\hline $\mathrm{BD} *(1)$ & $\pi^{*}$ & $\begin{array}{c}76 \% \mathrm{U}(f d)+24 \% \\
\mathrm{O}_{\mathrm{yl}}(p)\end{array}$ & 0.02 & $\begin{array}{c}79 \% \mathrm{O}_{\mathrm{yl}}(p)+21 \% \\
\mathrm{U}(f d)\end{array}$ & 0.06 & $\begin{array}{c}79 \% \mathrm{U}(f d)+21 \% \\
\mathrm{O}_{\mathrm{yl}}(p)\end{array}$ & 0.03 \\
\hline $\mathrm{BD} *(2)$ & $\pi^{*}$ & $\begin{array}{c}76 \% \mathrm{U}(f d)+24 \% \\
\mathrm{O}_{\mathrm{yl}}(p)\end{array}$ & 0.02 & $\begin{array}{c}79 \% \mathrm{O}_{\mathrm{yl}}(p)+21 \% \\
\mathrm{U}(f d)\end{array}$ & 0.06 & $\begin{array}{c}80 \% \mathrm{U}(f d)+20 \% \\
\mathrm{O}_{\mathrm{yl}}(p)\end{array}$ & 0.06 \\
\hline $\mathrm{BD} *(3)$ & $\sigma^{*}$ & $\begin{array}{c}64 \% \mathrm{U}(f d)+36 \% \\
\mathrm{O}_{\mathrm{yl}}\left(s p^{3}\right)\end{array}$ & 0.13 & $\begin{array}{c}73 \% \mathrm{O}_{\mathrm{yl}}\left(s p^{3}\right)+27 \% \\
\mathrm{U}(f d)\end{array}$ & 0.17 & $\begin{array}{c}73 \% \mathrm{U}(f d)+27 \% \\
\mathrm{O}_{\mathrm{yl}}\left(s p^{3}\right)\end{array}$ & 0.17 \\
\hline $\mathrm{BD} *(4)$ & $\pi^{*}$ & $\begin{array}{c}76 \% \mathrm{U}(f d)+24 \% \\
\mathrm{O}_{\mathrm{yl}}(p)\end{array}$ & 0.02 & $\begin{array}{c}79 \% \mathrm{O}_{\mathrm{yl}}(p)+21 \% \\
\mathrm{U}(f d)\end{array}$ & 0.05 & $\begin{array}{c}80 \% \mathrm{U}(f d)+20 \% \\
\mathrm{O}_{\mathrm{yl} 1}(p)\end{array}$ & 0.04 \\
\hline $\mathrm{BD} *(5)$ & $\pi^{*}$ & $\begin{array}{c}76 \% \mathrm{U}(f d)+24 \% \\
\mathrm{O}_{\mathrm{yl}}(p)\end{array}$ & 0.02 & $\begin{array}{c}79 \% \mathrm{O}_{\mathrm{yl}}(p)+21 \% \\
\mathrm{U}(f d)\end{array}$ & 0.06 & $\begin{array}{c}79 \% \mathrm{U}(f d)+21 \% \\
\mathrm{O}_{\mathrm{yl}}(p)\end{array}$ & 0.05 \\
\hline $\mathrm{BD} *(6)$ & $\sigma^{*}$ & $\begin{array}{c}64 \% \mathrm{U}(f d)+36 \% \\
\mathrm{O}_{\mathrm{yl}}\left(s p^{3}\right)\end{array}$ & 0.13 & $\begin{array}{c}73 \% \mathrm{O}_{\mathrm{yl}}\left(s p^{3}\right)+27 \% \\
\mathrm{U}(f d)\end{array}$ & 0.19 & $\begin{array}{c}75 \% \mathrm{U}(f d)+25 \% \\
\mathrm{O}_{\mathrm{yl}}\left(s p^{3}\right)\end{array}$ & 0.16 \\
\hline
\end{tabular}


Table S5. QTAIM metrics derived from SO-PT2 $(6,10)$ wave functions. The corresponding units (in parenthesis) are $\rho\left(\mathrm{e} / \mathrm{Bohr}^{3}\right), \mathrm{G}\left(\mathrm{kJ} / \mathrm{mol} \mathrm{Bohr}{ }^{3}\right), \mathrm{V}\left(\mathrm{kJ} / \mathrm{mol} \mathrm{Bohr}{ }^{3}\right), \mathrm{H}\left(\mathrm{kJ} / \mathrm{mol} \mathrm{Bohr}{ }^{3}\right)$, $\mathrm{H} / \rho(\mathrm{kJ} / \mathrm{mol}$ per electron)

\begin{tabular}{|c|c|c|c|c|c|c|c|}
\hline & & $\rho(\mathbf{r})$ & G(r) & $\mathbf{V}(\mathbf{r})$ & $|\mathbf{V}(\mathbf{r})| / \mathbf{G}(\mathbf{r})$ & $\mathbf{H}(\mathbf{r})$ & $\mathbf{H}(\mathbf{r}) / \rho(\mathbf{r})$ \\
\hline $\mathrm{UO}_{2}{ }^{2+}$ & $\mathrm{U} \equiv \mathrm{O}_{\mathrm{yl}}$ & 0.349 & 1135.9 & -2064.7 & 1.818 & -928.7 & -2663.4 \\
\hline \multirow{6}{*}{$\mathrm{UO}_{2} \mathrm{Cl}_{2}\left(\mathrm{H}_{2} \mathrm{~L}\right)$} & $\mathrm{U} \equiv \mathrm{O}_{\mathrm{yl}}$ & 0.310 & 958.4 & -1733.7 & 1.809 & -775.3 & -2498.6 \\
\hline & $\mathrm{U} \equiv \mathrm{O}_{\mathrm{yl}}$ & 0.312 & 961.2 & -1718.9 & 1.788 & -757.6 & -2426.8 \\
\hline & $\mathrm{U}-\mathrm{O}_{\mathrm{L}}$ & 0.079 & 227.5 & -258.2 & 1.135 & -30.7 & -388.8 \\
\hline & $\mathrm{U}-\mathrm{O}_{\mathrm{L}}$ & 0.081 & 231.1 & -264.7 & 1.145 & -33.5 & -415.6 \\
\hline & $\mathrm{U}-\mathrm{Cl}$ & 0.062 & 121.4 & -151.3 & 1.246 & -29.9 & -485.4 \\
\hline & $\mathrm{U}-\mathrm{Cl}$ & 0.063 & 124.3 & -155.0 & 1.247 & -30.7 & -490.9 \\
\hline \multirow{7}{*}{$\mathrm{UO}_{2} \mathbf{L}(\mathrm{MeOH})$} & $\mathrm{U} \equiv \mathrm{O}_{\mathrm{yl}}$ & 0.301 & 915.7 & -1622.9 & 1.772 & -707.2 & -2350.3 \\
\hline & $\mathrm{U} \equiv \mathrm{O}_{\mathrm{yl}}$ & 0.302 & 911.0 & -1621.9 & 1.780 & -710.8 & -2356.1 \\
\hline & $\mathrm{U}-\mathrm{O}_{\mathrm{L}}$ & 0.094 & 263.6 & -316.9 & 1.202 & -53.3 & -565.8 \\
\hline & $\mathrm{U}-\mathrm{O}_{\mathrm{L}}$ & 0.079 & 207.7 & -242.8 & 1.169 & -35.1 & -446.6 \\
\hline & $\mathrm{U}-\mathrm{O}_{\mathrm{MeOH}}$ & 0.053 & 143.5 & -152.1 & 1.060 & -8.6 & -161.9 \\
\hline & $\mathrm{U}-\mathrm{N}_{\mathrm{L}}$ & 0.059 & 122.7 & -143.3 & 1.167 & -20.5 & -347.0 \\
\hline & $\mathrm{U}-\mathrm{N}_{\mathrm{L}}$ & 0.055 & 113.1 & -130.5 & 1.154 & -17.4 & -315.0 \\
\hline
\end{tabular}




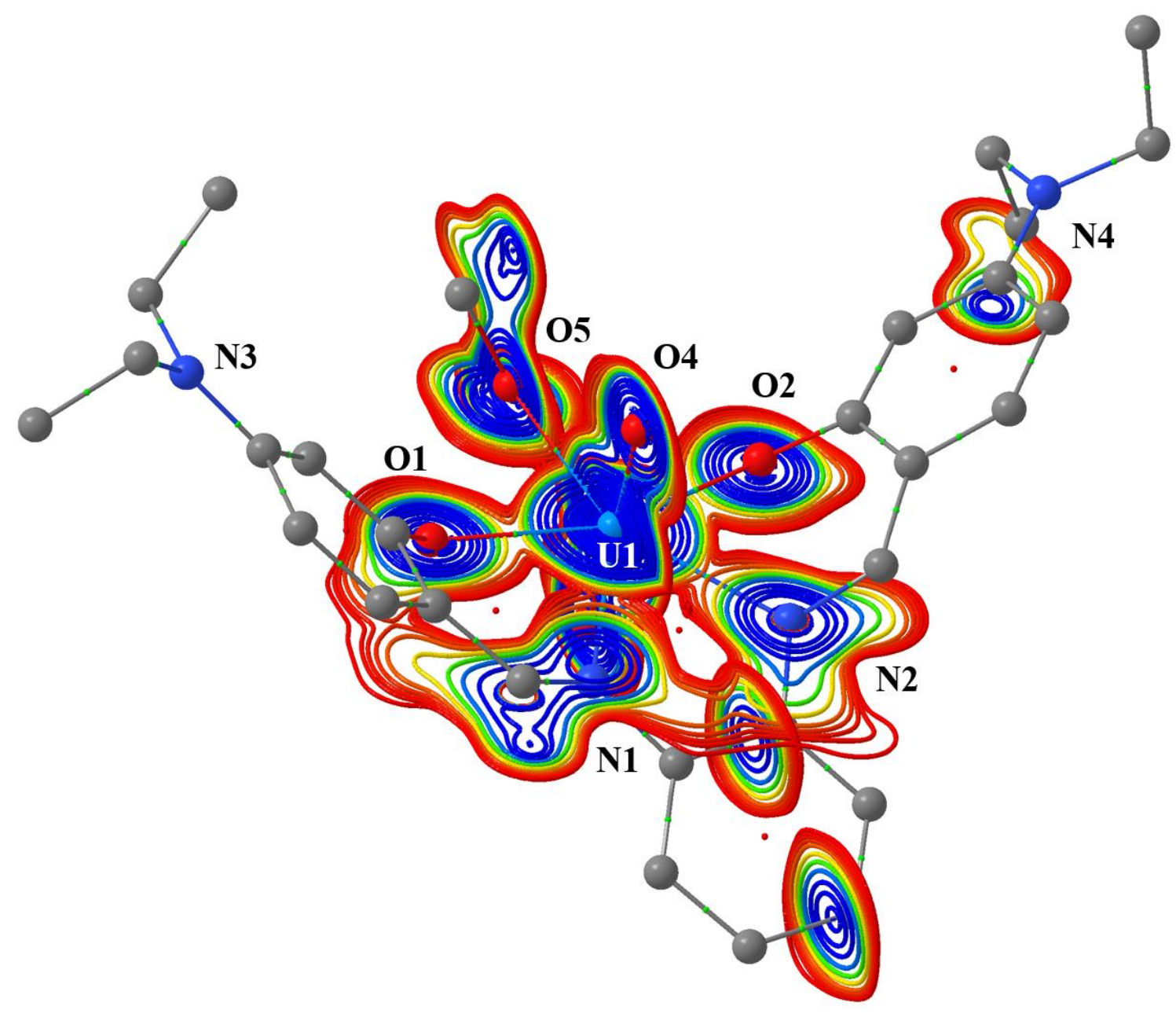

Figure S14. 2D contour map of the total energy density, $\mathrm{H}(\mathrm{r})$, in $\mathbf{U O}_{2} \mathbf{L}(\mathbf{M e O H})$. Isovalues range -0.09 (blue) to 0.00 (red). Blue regions indicate areas of increased covalent character.

Table S6. Covalency degree (kJ/mol per electron) values in axial and equatorial contributions.

\begin{tabular}{cccc}
\hline Covalency Degree & Axial & Equatorial & Total \\
\hline $\mathbf{U O}_{2}{ }^{2+}$ & -5326.8 & - & -5326.8 \\
$\mathbf{U} \mathbf{O}_{2} \mathbf{C l}_{2}\left(\mathbf{H}_{2} \mathbf{L}\right)$ & -4925.4 & -1780.7 & -6706.1 \\
$\mathbf{U O} \mathbf{O}_{2} \mathbf{L}(\mathbf{M e O H})$ & -4706.4 & -1836.2 & -6542.6 \\
\hline
\end{tabular}




\section{Crystallographic Details}

Table S7. Crystallographic information for $\mathrm{UO}_{2} \mathbf{L}(\mathrm{MeOH})$ and $\mathrm{UO}_{2} \mathrm{Cl}_{2}\left(\mathrm{H}_{2} \mathrm{~L}\right)$

\begin{tabular}{|c|c|c|}
\hline Compound & $\mathrm{UO}_{2} \mathrm{~L}(\mathrm{MeOH})$ & $\mathrm{UO}_{2} \mathrm{Cl}_{2}\left(\mathrm{H}_{2} \mathrm{~L}\right)$ \\
\hline Empirical Formula & $\mathrm{C}_{29} \mathrm{H}_{36} \mathrm{~N}_{4} \mathrm{O}_{5} \mathrm{U}$ & $\mathrm{C}_{28} \mathrm{H}_{34} \mathrm{Cl}_{2} \mathrm{~N}_{4} \mathrm{O}_{4} \mathrm{U}$ \\
\hline Formula Weight & 758.65 & 799.52 \\
\hline Color & Red & Orange \\
\hline Habit & Plate & Plate \\
\hline Temperature (K) & $150(1)$ & $150(1)$ \\
\hline Crystal System & Monoclinic & Triclinic \\
\hline Space Group & $\mathrm{P} 2{ }_{1} / c$ & $\mathrm{P} \overline{1}$ \\
\hline$a(\AA)$ & $14.5672(14)$ & $9.4250(11)$ \\
\hline$b(\AA)$ & $10.0842(10)$ & $11.8107(14)$ \\
\hline$c(\AA)$ & $19.3197(18)$ & $15.4884(18)$ \\
\hline$\alpha(\operatorname{deg})$ & 90 & $110.159(4)$ \\
\hline$\beta(\operatorname{deg})$ & $99.197(3)$ & $95.049(4)$ \\
\hline$\gamma(\mathrm{deg})$ & 90 & 99.191(3) \\
\hline Volume $\left(\AA^{3}\right)$ & $2801.6(5)$ & $1578.6(3)$ \\
\hline $\mathbf{Z}$ & 4 & 2 \\
\hline$\rho_{\text {calcd }}\left(\mathrm{Mg} / \mathrm{m}^{3}\right)$ & 1.799 & 1.682 \\
\hline$\mu\left(\mathrm{mm}^{-1}\right)$ & 5.839 & 5.348 \\
\hline GOF F $^{2}$ & 1.058 & 1.070 \\
\hline $\mathrm{R}^{a}(I>2.0 \sigma(\mathrm{I}))$ & 0.0236 & 0.0271 \\
\hline wR2 (all data) & 0.0477 & 0.0505 \\
\hline
\end{tabular}

a Definitions: R1 $=\Sigma|| F_{\mathrm{o}}|-| F_{\mathrm{c}}|/ / \Sigma| F_{\mathrm{o}} \mid, \mathrm{wR} 2=\left[\Sigma w\left(F_{\mathrm{o}}{ }^{2}-F_{\mathrm{c}}{ }^{2}\right)^{2} / \Sigma w\left(F_{\mathrm{o}}{ }^{2}\right)^{1 / 2}\right.$.

Goof $=\mathrm{S}=\left[\Sigma\left[\mathrm{w}\left(\mathrm{F}_{\mathrm{o}}^{2}-\mathrm{F}_{\mathrm{c}}^{2}\right)^{2}\right] /(\mathrm{n}-\mathrm{p})\right]^{1 / 2}$ where $\mathrm{n}$ is the number of reflections and $\mathrm{p}$ is the total number of parameters refined. 
Table S8. Relevant Bond lengths $[\AA]$ and angles $\left[{ }^{\circ}\right]$ for $\mathbf{U} \mathbf{O}_{2} \mathbf{L}(\mathbf{M e O H})$

\begin{tabular}{llll}
\hline $\mathrm{U} 1-\mathrm{O} 2$ & $2.3261(14)$ & $\mathrm{N} 1-\mathrm{U} 1-\mathrm{N} 2$ & $63.15(6)$ \\
$\mathrm{U} 1-\mathrm{O} 1$ & $2.2431(15)$ & $\mathrm{O} 5-\mathrm{U} 1-\mathrm{N} 2$ & $143.02(5)$ \\
$\mathrm{U} 1-\mathrm{N} 2$ & $\mathrm{O} 5-\mathrm{U} 1-\mathrm{N} 1$ & $153.54(5)$ \\
$\mathrm{U} 1-\mathrm{N} 1$ & $\mathrm{O} 3-\mathrm{U} 1-\mathrm{O} 2$ & $90.67(6)$ \\
$\mathrm{U} 1-\mathrm{O} 5$ & $2.5258(17)$ & $\mathrm{O} 3-\mathrm{U} 1-\mathrm{O} 1$ & $89.93(7)$ \\
$\mathrm{U} 1-\mathrm{O} 3$ & $2.4692(14)$ & $\mathrm{O} 3-\mathrm{U} 1-\mathrm{N} 2$ & $90.82(7)$ \\
$\mathrm{U} 1-\mathrm{O} 4$ & $1.7833(15)$ & $\mathrm{O} 3-\mathrm{U} 1-\mathrm{N} 1$ & $95.21(7)$ \\
$\mathrm{O} 2-\mathrm{U} 1-\mathrm{N} 2$ & $1.7828(15)$ & $\mathrm{O} 3-\mathrm{U} 1-\mathrm{O} 5$ & $89.23(6)$ \\
$\mathrm{O} 2-\mathrm{U} 1-\mathrm{N} 1$ & & $\mathrm{O} 4-\mathrm{U} 1-\mathrm{O} 2$ & $88.84(6)$ \\
$\mathrm{O} 2-\mathrm{U} 1-\mathrm{O} 5$ & $132.39(5)$ & $\mathrm{O} 4-\mathrm{U} 1-\mathrm{O} 1$ & $90.14(7)$ \\
$\mathrm{O} 1-\mathrm{U} 1-\mathrm{O} 2$ & $73.44(5)$ & $\mathrm{O} 4-\mathrm{U} 1-\mathrm{N} 2$ & $89.91(7)$ \\
$\mathrm{O} 1-\mathrm{U} 1-\mathrm{N} 2$ & $157.01(6)$ & $\mathrm{O} 4-\mathrm{U} 1-\mathrm{N} 1$ & $85.82(7)$ \\
$\mathrm{O} 1-\mathrm{U} 1-\mathrm{N} 1$ & $133.40(6)$ & $\mathrm{O} 4-\mathrm{U} 1-\mathrm{O} 5$ & $89.71(6)$ \\
$\mathrm{O} 1-\mathrm{U} 1-\mathrm{O} 5$ & $70.38(6)$ & $\mathrm{O} 4-\mathrm{U} 1-\mathrm{O} 3$ & $178.92(7)$ \\
& $83.58(6)$ & &
\end{tabular}

Table S9. Relevant Bond lengths $[\AA]$ and angles $\left[{ }^{\circ}\right]$ for $\mathbf{U O}_{2} \mathbf{C l}_{2}\left(\mathbf{H}_{2} \mathbf{L}\right)$

\begin{tabular}{llll}
\hline $\mathrm{U} 1-\mathrm{O} 3$ & $1.7679(16)$ & $\mathrm{O} 3-\mathrm{U} 1-\mathrm{Cl} 2$ & $88.20(6)$ \\
$\mathrm{U} 1-\mathrm{O} 1$ & $2.2964(15)$ & $\mathrm{O} 1-\mathrm{U} 1-\mathrm{Cl}$ & $89.40(4)$ \\
$\mathrm{U} 1-\mathrm{O} 4$ & $1.7691(17)$ & $\mathrm{O} 1-\mathrm{U} 1-\mathrm{Cl}$ & $169.44(4)$ \\
$\mathrm{U} 1-\mathrm{O} 2$ & $2.2915(14)$ & $\mathrm{O} 4-\mathrm{U} 1-\mathrm{O} 1$ & $91.96(8)$ \\
$\mathrm{U} 1-\mathrm{C} 11$ & $2.6823(6)$ & $\mathrm{O} 4-\mathrm{U} 1-\mathrm{O} 2$ & $91.04(7)$ \\
$\mathrm{U} 1-\mathrm{C} 12$ & $2.6735(7)$ & $\mathrm{O} 4-\mathrm{U} 1-\mathrm{Cl}$ & $90.46(6)$ \\
$\mathrm{O} 3-\mathrm{U} 1-\mathrm{O} 1$ & $86.96(7)$ & $\mathrm{O} 4-\mathrm{U} 1-\mathrm{Cl}$ & $92.75(7)$ \\
$\mathrm{O} 3-\mathrm{U} 1-\mathrm{O} 4$ & $178.70(8)$ & $\mathrm{O} 2-\mathrm{U} 1-\mathrm{O} 1$ & $82.01(5)$ \\
$\mathrm{O} 3-\mathrm{U} 1-\mathrm{O} 2$ & $88.09(6)$ & $\mathrm{O} 2-\mathrm{U} 1-\mathrm{Cl}$ & $171.32(4)$ \\
$\mathrm{O} 3-\mathrm{U} 1-\mathrm{Cl1}$ & $90.26(5)$ & $\mathrm{O} 2-\mathrm{U} 1-\mathrm{Cl} 2$ & $88.46(4)$ \\
& & $\mathrm{Cl} 2-\mathrm{U} 1-\mathrm{Cl}$ & $100.01(2)$
\end{tabular}


